\title{
Sequence and gene content of a large fragment of a lizard sex chromosome and evaluation of candidate sex differentiating gene R-spondin 1
}

\author{
Tariq Ezaz ${ }^{1 *}$, Bhumika Azad ${ }^{1}$, Denis O'Meally ${ }^{1}$, Matthew J Young ${ }^{1}$, Kazumi Matsubara ${ }^{1}$, Melanie J Edwards ${ }^{1}$, \\ Xiuwen Zhang ${ }^{1}$, Clare E Holleley ${ }^{1}$, Janine E Deakin', Jennifer A Marshall Graves ${ }^{1,2}$, Arthur Georges ${ }^{1}$, \\ Scott V Edwards ${ }^{3}$ and Stephen D Sarre ${ }^{1}$
}

\begin{abstract}
Background: Scant genomic information from non-avian reptile sex chromosomes is available, and for only a few lizards, several snakes and one turtle species, and it represents only a small fraction of the total sex chromosome sequences in these species.

Results: We report a $352 \mathrm{~kb}$ of contiguous sequence from the sex chromosome of a squamate reptile, Pogona vitticeps, with a ZZ/ZW sex microchromosome system. This contig contains five protein coding genes (oprd1, rccl, znf91, znf131, znf180), and major families of repetitive sequences with a high number of copies of LTR and non-LTR retrotransposons, including the CR1 and Bov-B LINEs. The two genes, oprd1 and rccl are part of a homologous syntenic block, which is conserved among amniotes. While oprd1 and rccl have no known function in sex determination or differentiation in amniotes, this homologous syntenic block in mammals and chicken also contains $R$-spondin 1 (rspo1), the ovarian differentiating gene in mammals. In order to explore the probability that rspo 1 is sex determining in dragon lizards, genomic BAC and CDNA clones were mapped using fluorescence in situ hybridisation. Their location on an autosomal microchromosome pair, not on the ZW sex microchromosomes, eliminates rspo 1 as a candidate sex determining gene in $P$. vitticeps.

Conclusion: Our study has characterized the largest contiguous stretch of physically mapped sex chromosome sequence $(352 \mathrm{~kb}$ ) from a ZZ/ZW lizard species. Although this region represents only a small fraction of the sex chromosomes of $P$. vitticeps, it has revealed several features typically associated with sex chromosomes including the accumulation of large blocks of repetitive sequences.
\end{abstract}

Keywords: ZW sex chromosomes, Genotypic sex determination (GSD), Temperature dependent sex determination (TSD), RSPO1, Squamata, Reptilia

\section{Background}

Chromosomes are remarkably conserved across vertebrates, showing striking conservation of gene content and gene order of substantial regions of synteny over millions of years. For example, the karyotypes of different bird species are extremely similar [1] and comparative chromosome painting of squamate reptiles (lizards

\footnotetext{
* Correspondence: Tariq.Ezaz@canberra.edu.au

'Institute for Applied Ecology, University of Canberra, Canberra 2601, Australia

Full list of author information is available at the end of the article
}

and snakes) macrochromosomes shows ancestral homology dating back to the early Jurassic $[2,3]$.

In remarkable contrast to this deep conservation, and surprising, considering their critical role in reproduction, sex chromosomes show a lack of homology and frequent rearrangements and transitions between and even within lineages [4]. The most stable and conserved vertebrate systems are in therian mammals (eutherians and marsupial mammals with a highly conserved XX female: $\mathrm{XY}$ male system of male heterogamety), and birds and snakes (with highly conserved ZW female: ZZ male systems of female heterogamety). These three chromosome

\section{Ciomed Central}


systems, that is, $\mathrm{XY}$ of mammals, ZW of snakes and ZW of birds show no genetic homology and are thought to have evolved independently $[4,5]$. The evolutionary dynamism of sex chromosomes is more obvious from the distinct forms of XY and ZW chromosomes among vertebrates even within taxonomic families $[5,6]$. It is this lability and the role of the sex chromosomes in the fundamental trait of sex determination that makes non-mammalian vertebrates of extreme interest for studying the processes behind genome organization and evolution [5-8].

Sex chromosomes are generally thought to have evolved from a homologous autosomal pair, when one partner acquired a sex determining gene [9-11]. Accumulation of sexually advantageous mutations near the sex determining locus led to selection for suppression of recombination, which in turn accelerated the loss of active genes, deletions and insertions from the sex specific $\mathrm{Y}$ or W chromosomes [11-14]. The loss of genetic material and accumulation of repetitive sequences on the $\mathrm{Y}$ or $\mathrm{W}$ is obvious in many species as cytological heteromorphism. This process of repeat accumulation is rapid and stochastic, generating various intermediates between homomorphy and extreme differentiation in squamate reptiles (snakes and lizards), and variation in the gene content as can be seen in the Y chromosome of different mammals $[6,15]$.

Despite the discovery of XY and ZW sex chromosomes in the early $19^{\text {th }}$ century [16-18], few master sex determining genes have been identified. These include SRY in therian mammals $[19,20]$, and a variety of genes in fish and frog: $d m y$ in medaka (Oryzias latipes) [21,22], gsdf $(Y)$ in medaka (O. luzonensis) [23], dmw in clawed frog (Xenopus laevis) [24], sdy in rainbow trout (Oncorhynchus mykiss) [25], and amhy in Patagonian pejerrey (Odontesthes hatcheri) [26]. In all but three cases ( $s d y$ in rainbow trout, amhy in Patagonian pejerrey and $g s d f(Y)$ in medaka) $[23,25,26]$, master sex determining genes are transcription factors, in so far as they appear to act by up- or down-regulating transcription in the vertebrate sex differentiation pathway. This newly discovered diversity in regulation suggests that the de novo evolution of master sex determining genes from genes that are not involved in sex differentiation can occur in vertebrates. It occurs less frequently than those that are involved in the sexual differentiation pathway. However, due to recent advancement in molecular genetics and genome sequencing technologies, novel master sex determining genes are likely to be discovered at an accelerating rate, in turn discovering novel mechanisms of sex determination.

Reptiles are of particular interest because they display astonishing variation in the sex determining system. They are represented by species which display all types of chromosomal system (e.g. XY, ZW, including multiple sex chromosomes) and temperature-dependent sex determination (TSD), as well as species in which the two modes of sex determination interact $[6,8,27-29]$. This diversity is well established in the literature, so it is remarkable that little is known of the genes involved. Non-avian reptiles remain the only vertebrate group in which no master sex determining gene has been identified. They therefore promise to yield novel insights into vertebrate sex determination.

The best-studied sex chromosomes among non-avian reptiles are those of snakes [30-34]. Like birds, snakes have a $\mathrm{ZZ}$ male ZW female system, and $\mathrm{Z}$ gene content is conserved across all species so far examined. However, gene mapping shows that the sex chromosomes of snakes are not homologous to those of birds or mammals [31,32], nor to those of a ZW turtle (Pelodiscus sinensis), a ZW gecko (Gekko hokouensis), a ZW dragon lizard (Pogona vitticeps) or an XY green anole (Anolis carolinensis) [31,32,35-38]. Four genes that are Z-linked in G. hokouensis are autosomal in the dragon lizard Pogona vitticeps [35] and in snakes [32,37] and five genes that are Z-borne in snakes and birds are autosomal in the dragon lizard P. vitticeps [35]. Even within dragons (Agamidae), comparative mapping of a sexlinked marker shows that the ZW sex microchromosomes of three Australian agamid species (P. vitticeps, P. barbata and Diporiphora nobbi) are homologous, but their homology does not extend to a fourth species, Ctenophorus fordi $[29,39]$. Thus there is likely to be a number of independent origins of the sex chromosomes, not only between two families of lizards (Gekkonidae and Agamidae), but also among closely related species in the same subfamily.

The central bearded dragon, $P$. vitticeps, is a powerful model for the study of the evolution of sex determination because it has differentiated sex chromosomes [40], but phenotypic sex can be reversed by high temperatures, producing all female individuals [28]. Thus, sex in $P$. vitticeps is determined by the interplay between genotype and environment, producing genotype-phenotype discordant individuals. Discovering novel sex determining genes in this species will therefore broaden our understanding of how sex is genetically determined, as well as of how environment can regulate genetic machinery. This species has 16 pairs of chromosomes -6 pairs are macrochromosomes and 10 pairs are microchromosomes [41] with female heterogamety (ZZ male, ZW female) [40]. The sex chromosomes are microchromosomes and the W chromosome is larger than the $\mathrm{Z}$ [42]. The W chromosome is also highly heterochromatic [40] which suggests that its sequence content is different from the $\mathrm{Z}$ and that an amplification of repetitive DNA is likely to have occurred during its evolution. 
We previously reported the isolation of $\mathrm{Z}$ and $\mathrm{W}$ linked markers, developed a genetic sex test and sequenced a $3.2 \mathrm{~kb}$ region of the sex chromosomes [28,29] in Pogona vitticeps. In this study, we extend the sequenced region of the $P$. vitticeps sex microchromosomes to $352 \mathrm{~kb}$ using a BAC walking approach. We report 28 open reading frame (ORFs) including three gene family members (oprd, rcc and $z n f$ ), and eight major groups of repetitive elements. Comparative analysis of this partial sex chromosome sequence, allowed us to identify additional potential candidate sex chromosome genes in $P$. vitticeps. One of these genes, $R$-spondin 1 (rspo1), has a central role in vertebrate ovarian differentiation [43-45] and we evaluate it as a candidate sex-determining gene in $P$. vitticeps.

\section{Results}

Isolation and physical mapping of Pogona vitticeps sex chromosome BAC clones

Ten BAC clones were isolated by genomic library screening with previously identified sex linked sequences $[27,28]$ and chromosome walking. We sequenced five of the 10 BAC clones, which ranged in length from $98 \mathrm{~kb}$ to $202 \mathrm{~kb}$ (GenBank Accessions: KF541650; KF541651; KF541652; KF541653; KF541655, Table 1), while the remaining five $\mathrm{BAC}$ clones overlapped the sequenced clones. Reads from all five BAC clones were assembled into a single contiguous sequence of $352 \mathrm{~kb}$ (GenBank Accession: KF541658). Analysis of this partial sex chromosome sequence revealed that the contig contains the previously identified W marker Pv72W and contig C1, but not the Z marker Pv71Z [27,28] (Figure 1a). This implies that the contig is likely to have W chromosomal origin and the contig therefore represents partial W sequence (Figure 1a). The BAC clones were physically mapped by DNA FISH as well as by fiber FISH to verify their location on the sex chromosomes (Figure 1b, e). The intensity and pattern of hybridisation signals of two colour DNA FISH with BAC clones Pv151_P16 and Pv151_D05 were different between $\mathrm{Z}$ and $\mathrm{W}$ chromosomes. Primary hybridisation of the BAC clones was to the $\mathrm{Z}$ and $\mathrm{W}$ chromosomes, with signal on the $\mathrm{W}$ chromosome more intense than that on the $\mathrm{Z}$ chromosome (Figure 1b). Terminal hybridisation signals on the long arm of chromosome 2 suggested these BAC clones may share some repetitive elements with the NORassociated heterochromatin (Figure 1b, [42]).

Two of the largest BAC clones, Pv151_P16 (about $182 \mathrm{~kb}$ ) and Pv237_P23, (about $202 \mathrm{~kb}$ ), which represent the two ends of the $352 \mathrm{~kb}$ contig and overlap in the middle, showed green, orange and yellow (overlapping) fluorescent signals on chromatin fibers (Figure 1e) confirming our physical map of BAC clones (Figure 1b). The observation of multiple regions of green and orange on a single fiber suggests repetition of sequences contained within the BAC outside the contig (Figure 1e). As a control for comparative analysis between sex chromosome and autosome sequences, substantial genomic sequences were also obtained from three autosomal BAC clones (Table 1).

\section{Sequence analysis of the partial sex chromosome contig}

We used Genscan [46] to analyze sequence of this contig. This identified the contig as an isochore type 2 with $44.5 \%$ GC content. Genscan also predicted 28 ORFs (Table 2). They ranged between 57 amino acids (aa) to 3194 aa (Table 2). BLAST (blastp, Anolis carolonensis; [47]) analysis of these ORFs supported the predictions for the proteincoding genes, opioid receptor, delta 1 (oprd1) and regulator of chromosome condensation 1 ( $r c c 1$ ) and three zinc finger protein genes, $z n f 91$, znf135 and $z n f 180$ (Table 2).

The repetitive sequence content of sex chromosomes was examined using Repbase-GIRI [48]. Analysis of repetitive sequences of the sex chromosome BAC clones revealed the highly repetitive nature of $P$. vitticeps sex chromosome sequences (Figure 1c and d, Table 1, Additional file 1). For example, one of the smallest sex chromosome BAC clones, Pv03_L07 (about 98 kb) contains $41 \%$ repetitive sequences of which $43 \%$ are non-LTR retrotransposons (Table 1). Overall, LTR and non-LTR retrotransposons constituted the bulk of the repetitive sequences of the sex chromosome BAC clones. We compared this composition with that of three autosomal BAC contigs. In general, sex chromosome BAC clones have three times more repetitive sequences (Table 1). Autosomal BAC clones showed no preferential accumulation of any specific repetitive sequences (Table 1).

We also investigated the accumulation of microsatellite repeats within the $352 \mathrm{~kb}$ sex linked contig using Msat commander [49]. Our analysis identified 16 STR loci consisting of three di-nucleotide (AC, AG, AT), three trinucleotide (AAC, AGC, AAT), eight tetra-nucleotide (AAGG, AATC, AATG, ACGC, AAAC, ATCC, AGAT, AAAT), and two penta-nucleotide (AAAAT, AAATC) repeat motifs within the $352 \mathrm{~kb}$ sex linked contig. At least five of these loci were found to be amplified on the W chromosomes (data not shown, will be published elsewhere). These loci therefore provide opportunities to develop sex-linked markers.

\section{Comparative analysis of the sequenced region}

Comparative orthology analysis revealed that orthologues of oprd1 and rcc1 are part of a conserved homologous syntenic block (HSBs) in other vertebrates (e.g. chicken, chromosome 23; human chromosome 1). This region is not on the sex chromosomes of these other species, confirming the independent origin of the dragon sex chromosomes.

Analysis of genes in this HSB identified a candidate sex determining gene for $P$. vitticeps, the mammalian 
Table 1 A comparison of the major classes of repetitive sequences in sex chromosomal and autosomal BAC clones in Pogona vitticeps

Sex chromosome BAC clones

Autosome BAC clone

\begin{tabular}{|c|c|c|c|c|c|c|c|c|c|c|c|c|c|c|c|c|}
\hline \multirow{2}{*}{ Repeat class } & \multicolumn{2}{|c|}{ Pv03_L07 (98 kb) } & \multicolumn{2}{|c|}{ Pv151_P16 (183 Kb) } & \multicolumn{2}{|c|}{ Pv159_B13 (113 Kb) } & \multicolumn{2}{|c|}{ Pv237_P23 (202 kb) } & \multicolumn{2}{|c|}{ Pv151_D05 (156 Kb) } & \multicolumn{2}{|c|}{ Pv176_G09 (145 Kb) } & \multicolumn{2}{|c|}{ Pv100_013 (139 kb) } & \multicolumn{2}{|c|}{ Pv215_D15 (134 Kb) } \\
\hline & Fragments & $\begin{array}{l}\text { Size } \\
\text { (bp) }\end{array}$ & Fragments & $\begin{array}{l}\text { Size } \\
\text { (bp) }\end{array}$ & Fragments & $\begin{array}{l}\text { Size } \\
\text { (bp) }\end{array}$ & Fragments & $\begin{array}{l}\text { Size } \\
\text { (bp) }\end{array}$ & Fragments & $\begin{array}{l}\text { Size } \\
\text { (bp) }\end{array}$ & Fragments & $\begin{array}{l}\text { Size } \\
\text { (bp) }\end{array}$ & Fragments & $\begin{array}{l}\text { Size } \\
\text { (bp) }\end{array}$ & Fragments & $\begin{array}{l}\text { Size } \\
\text { (bp) }\end{array}$ \\
\hline Integrated virus & 0 & 0 & 0 & 0 & 1 & 47 & 1 & 47 & 0 & 0 & 0 & 0 & 0 & 0 & 0 & 0 \\
\hline $\begin{array}{l}\text { Interspersed } \\
\text { repeat }\end{array}$ & 1 & 49 & 3 & 254 & 2 & 126 & 1 & 49 & 1 & 77 & 2 & 165 & 0 & 0 & 0 & 0 \\
\hline DNA transposon & 30 & 2872 & 53 & 4050 & 37 & 3250 & 69 & 5288 & 58 & 4343 & 58 & 5283 & 49 & 6140 & 40 & 4906 \\
\hline $\begin{array}{l}\text { Endogenous } \\
\text { retrovirus }\end{array}$ & 23 & 5252 & 39 & 6836 & 9 & 489 & 16 & 1036 & 11 & 726 & 25 & 1888 & 16 & 1132 & 14 & 990 \\
\hline LTR transposon & 37 & 12385 & 87 & 25553 & 48 & 9078 & 82 & 12342 & 61 & 5673 & 44 & 3613 & 66 & 4350 & 71 & 5287 \\
\hline $\begin{array}{l}\text { Non-LTR } \\
\text { transposon }\end{array}$ & 58 & 17770 & 101 & 28645 & 74 & 17366 & 148 & 46284 & 121 & 40003 & 46 & 5767 & 51 & 5000 & 54 & 5448 \\
\hline Pseudogene & 0 & 0 & 0 & & 0 & 0 & 1 & 68 & 1 & 68 & 2 & 173 & 0 & 0 & 0 & 0 \\
\hline Simple repeat & 21 & 2189 & 35 & 3743 & 6 & 597 & 37 & 3710 & 29 & 2941 & 0 & 0 & 2 & 137 & 2 & 133 \\
\hline Total & 170 & 40517 & 318 & 69081 & 177 & 30953 & 355 & 68824 & 282 & 53831 & 177 & 16889 & 184 & 16759 & 181 & 16764 \\
\hline Proportion (\%) & & 41.3439 & & 37.74918 & & 27.392 & & 34.0713 & & 34.5071 & & 11.6476 & & 13.9658 & & 10.4775 \\
\hline
\end{tabular}




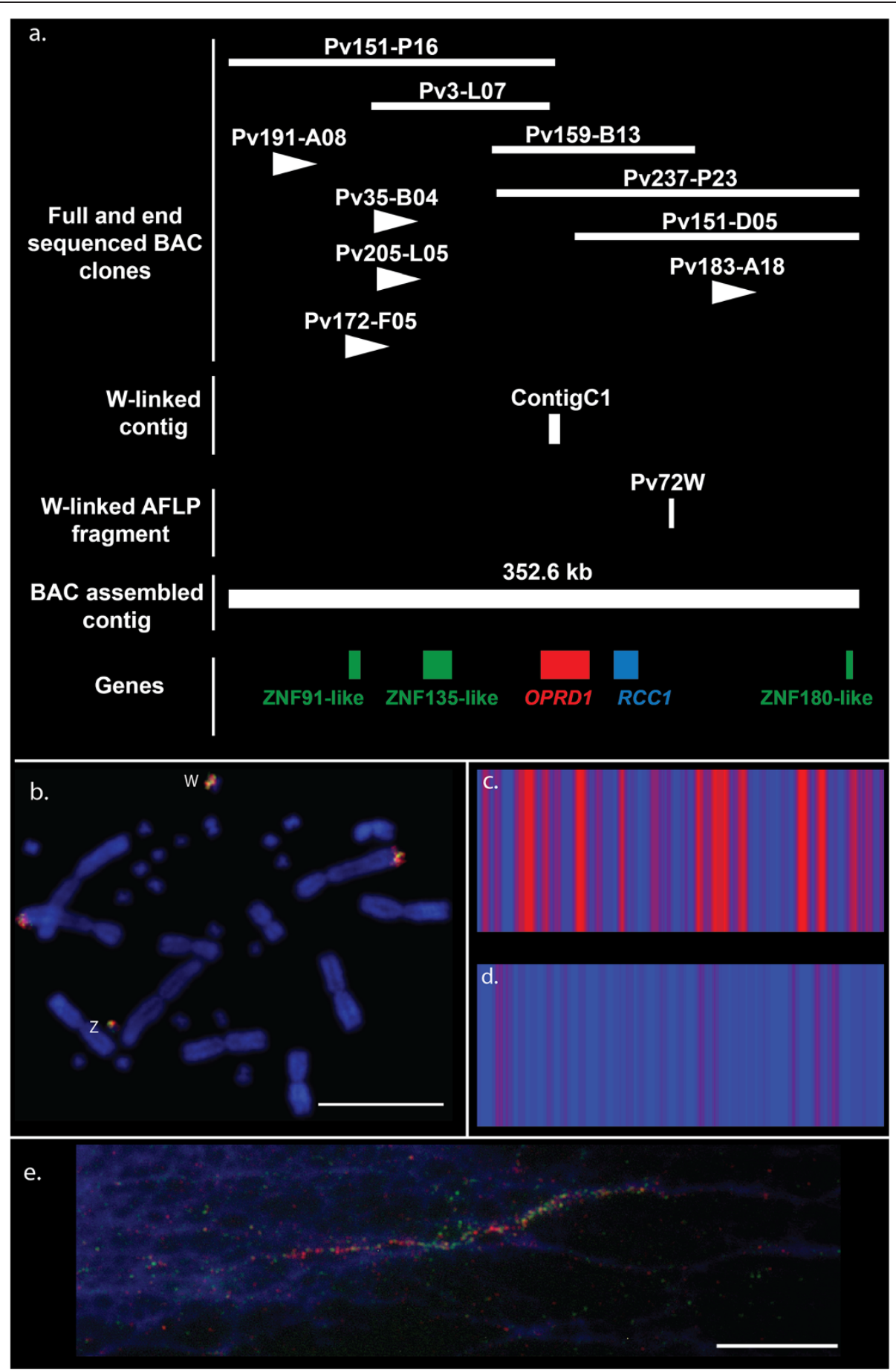

Figure 1 Isolation and physical mapping of sex chromosome BAC clones in Pogona vitticeps. a: schematic representation of $352 \mathrm{~kb}$ partial sex chromosome contig showing relative locations (not to scale) of fully and end sequenced BAC clones, previously identified W-linked contig and AFLP marker, proteins and genes; $\mathbf{b}$ : two colour FISH showing locations of two sex chromosome BAC clones at either end of the $P$. vitticeps $352 \mathrm{~kb}$ contig (Pv151_P16 green and Pv151_D05 orange; yellow denotes overlapping signals). Hybridization signal differences between Z and W chromosomes are visible; c: scalable vector graphics (SVG) plot (generated by Repbase-GIRI [48]) of sex chromosome BAC clone Pv03_L07, high density and frequency of red vertical bars represent distributions and locations of repetitive sequences; d: SVG plot of autosomal BAC clone Pv176_G09 showing low frequencies of red vertical bars indicating accumulation of low number of repeats compared to that of the sex chromosome BAC clone; e: two colour fiber FISH (Pv151_P16 orange, Pv237_P23 green), showing orange and green regions and regions of overlap (yellow). Scale bars represent $10 \mu \mathrm{m}$. 
Table 2 Summary of Blastp (refseq Anolis carolinensis [47]) analysis of Genscan [46] predicted ORFs from 352 kb contig sequences in Pogona vitticeps

\begin{tabular}{|c|c|c|c|c|c|c|c|}
\hline & ORF & Predicted genes/proteins & Total & Query & E value & Max & $\begin{array}{l}\text { Accession } \\
\text { anolis }\end{array}$ \\
\hline No. & Length (aa) & & & & & & \\
\hline 1 & 776 & PREDICTED: receptor expression-enhancing protein 3-like & 94.4 & $12 \%$ & SE-21 & $55 \%$ & XP_003223495.1 \\
\hline 2 & 2208 & PREDICTED: hypothetical protein in LOC100567032 & 302 & $27 \%$ & $3 \mathrm{E}-82$ & $32 \%$ & XP_00322950.1 \\
\hline 3 & 576 & PREDICTED: protein FAM135-B like & 33.5 & $18 \%$ & 0.33 & $26 \%$ & XP_003219535.1 \\
\hline 4 & 718 & PREDICTED: zinc finger protein 91-like & 9847 & $83 \%$ & 0 & $78 \%$ & XP_003229097.1 \\
\hline 5 & 978 & $\begin{array}{c}\text { PREDICTED: retrotransposable element Tf2 } 155 \mathrm{kDa} \text { protein } \\
\text { type 1-like }\end{array}$ & 423 & $82 \%$ & $1 \mathrm{E}-83$ & $36 \%$ & XP_003224123.1 \\
\hline 6 & 92 & $\begin{array}{c}\text { PREDICTED: leucine-rich repeat and fibronectin type III } \\
\text { domain-containing protein }\end{array}$ & 27.7 & $54 \%$ & 0.65 & $34 \%$ & XP_003222916.1 \\
\hline 7 & 1575 & $\begin{array}{c}\text { PREDICTED: retrotransposable element Tf Tf2 } 155 \mathrm{kDa} \\
\text { protein type-like }\end{array}$ & 360 & $40 \%$ & $3 \mathrm{E}-79$ & $39 \%$ & XP_003220874.1 \\
\hline 8 & 340 & $\begin{array}{l}\text { PREDICTED: 1, 25-dihydroxivitamin D(3) 24-hydroxylase, } \\
\text { mitochondrial-like }\end{array}$ & 113 & $37 \%$ & $2 \mathrm{E}-27$ & $48 \%$ & XP_003228807.1 \\
\hline 9 & 1092 & PREDICTED: zinc finger protein 135-like & 3327 & $41 \%$ & 0 & $73 \%$ & XP_003229099.1 \\
\hline 10 & 1012 & PREDICTED: hypothetical protein LOC100556889 & 155 & $26 \%$ & 7E-39 & $34 \%$ & XP_003217306.1 \\
\hline 11 & 1009 & $\begin{array}{c}\text { PREDICTED: retrotransposable element Tf2 } 155 \mathrm{kDa} \text { protein } \\
\text { type 1-like }\end{array}$ & 364 & $65 \%$ & 7E-81 & $40 \%$ & XP_003220874.1 \\
\hline 12 & 204 & PREDICTED: serologically defined colon cancer antigen 8-like & 57 & $45 \%$ & $9 \mathrm{E}-10$ & $40 \%$ & XP_003225236.1 \\
\hline 13 & 298 & $\begin{array}{c}\text { PREDICTED: 1, } 25 \text {-dihydroxyvitamin D(3) 24-hydroxylase, } \\
\text { mitochondrial-like }\end{array}$ & 68.6 & $42 \%$ & $5 \mathrm{E}-13$ & $34 \%$ & XP_003228807.1 \\
\hline 14 & 573 & PREDICTED: hypothetical protein LOC100566709 & 253 & $43 \%$ & $5 E-73$ & $48 \%$ & XP_003227941.1 \\
\hline 15 & 297 & PREDICTED: RNA-binding protein with multiple splicing-like, partial & 31.2 & $8 \%$ & 0.41 & $56 \%$ & XP_003227406.1 \\
\hline 16 & 418 & PREDICTED: delta-type opioid receptor-like & 623 & $90 \%$ & 0 & $82 \%$ & XP_003229441.1 \\
\hline 17 & 118 & PREDICTED: leucine-rich repeat neuronal protein 2-like & 28.5 & $41 \%$ & 0.94 & $26 \%$ & XP_003220412.1 \\
\hline 18 & 125 & PREDICTED: ubiquitin-conjugating enzyme E2 E1-like isoform 1 & 29.3 & $52 \%$ & 0.42 & $28 \%$ & XP_003226269.1 \\
\hline 19 & 106 & $\begin{array}{l}\text { PREDICTED: LOW QUALITY PROTEIN: dynein heavy chain 17, } \\
\text { axonemal-like }\end{array}$ & 28.9 & $81 \%$ & 0.53 & $28 \%$ & XP_003217173.1 \\
\hline 20 & 424 & PREDICTED: regulator of chromosome condensation-like & 734 & $100 \%$ & 0 & $85 \%$ & XP_003229442.1 \\
\hline 21 & 748 & PREDICTED: ras-related protein Rab-17-like & 113 & $18 \%$ & $3 \mathrm{E}-27$ & $44 \%$ & XP_003215266.1 \\
\hline 22 & 57 & PREDICTED: class I histocompatibility antigen, F10 alpha chain-like & 28.1 & $50 \%$ & 0.21 & $31 \%$ & XP_003227795.1 \\
\hline 23 & 527 & PREDICTED: hypothetical protein LOC100561123 & 93.2 & $49 \%$ & $1 \mathrm{E}-19$ & $28 \%$ & XP_003229050.1 \\
\hline 24 & 253 & PREDICTED: excitatory amino acid transporter 2-like & 28.1 & $22 \%$ & 4.5 & $41 \%$ & XP_003226244.1 \\
\hline 25 & 3194 & PREDICTED: hypothetical protein LOC100561123 & 546 & $33 \%$ & $3 \mathrm{E}-39$ & $29 \%$ & XP_003225516.1 \\
\hline 26 & 358 & PREDICTED: ubiquitin carboxyl-terminal hydrolase 25 -like & 67.4 & $74 \%$ & $3 \mathrm{E}-12$ & $25 \%$ & XP_003219106.1 \\
\hline 27 & 573 & PREDICTED: hypothetical protein LOC100567032 & 53.9 & $14 \%$ & $2 \mathrm{E}-07$ & $33 \%$ & XP_003229050.1 \\
\hline 28 & 396 & PREDICTED: zinc finger protein 180-like, partial & 1130 & $44 \%$ & $3 \mathrm{E}-79$ & $72 \%$ & XP_003230299.1 \\
\hline
\end{tabular}

Only the top hits based on E-values are listed.

ovarian differentiation gene R-spondin 1(RSPO1). Since, we did not find rspol sequences within the sequenced region, we cloned and mapped the dragon rspol orthologue.

\section{Cloning and characterization of $r$ spo 1}

We screened the BAC library using primers designed from sequences obtained from a testis transcriptome (T. Ezaz unpublished). Using this approach we isolated two rspo1-containing BAC clones (Pv100_O13 and Pv215_D15; GenBank
Accessions: KF541656; KF541657). These BAC clones were annotated using Genscan [46], revealing 19 and 17 ORFs respectively (Additional file 2). Blastp [47] analysis of those ORFs identified 10 genes from the region surrounding rspo1 in other amniote species; Figure 2, Additional file 2), corresponding to the conserved HSB homologous to chicken chromosome 23 and human chromosome 1.

We also cloned cDNA of a homologue of rspol in $P$. vitticeps by PCR amplification of RNA using primers 


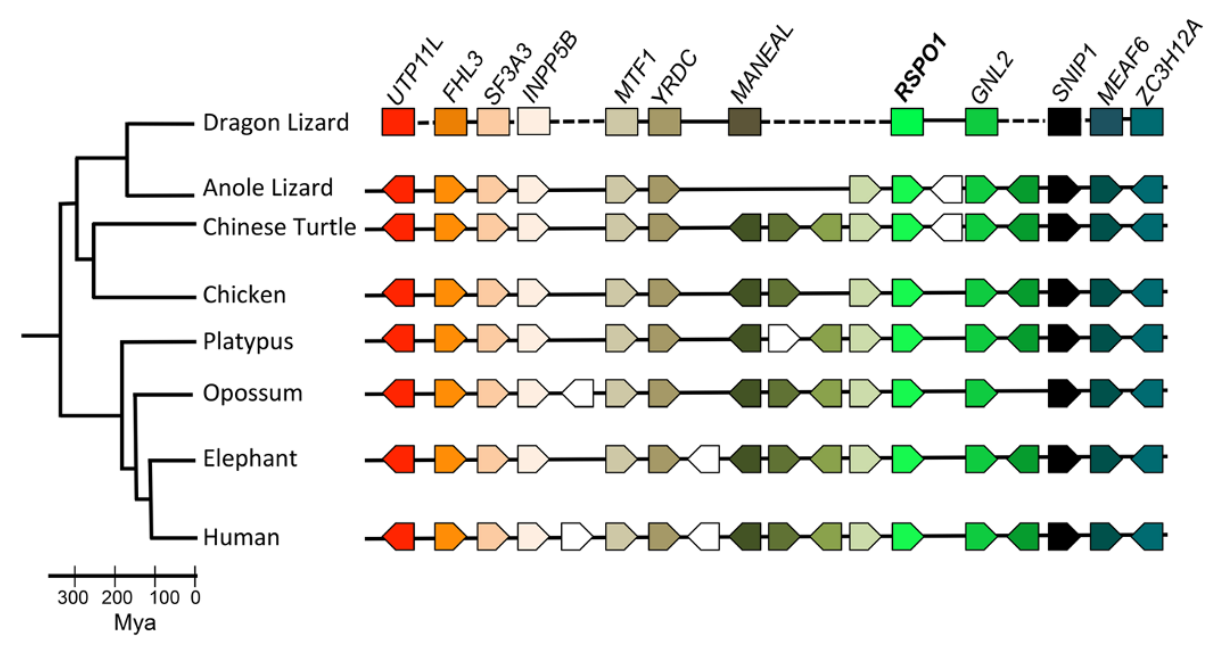

Figure 2 Gene content of the sequenced rspo1-containing BAC clones in the dragon lizard (Pogona vitticeps) is conserved when compared to other amniote species. Arrows indicate direction of transcription. Gene order and orientation is unknown for the dragon lizard due to gaps in BAC sequence. White arrows indicate genes with no orthologue in the region for the other species shown.

designed against $P$. vitticeps' testis transcriptome derived rspo1 (T. Ezaz unpublished). We sequenced three cDNA clones, finding consensus sequences of the two fragments 703 and 956 bp length which partially overlapped. The total length of the consensus sequence covered by the two fragments was $1347 \mathrm{bp}$.

To eliminate the possibility that rspo1 gene sequences reside on sex chromosomes in this species, we directly sequenced the PCR products from 10 phenotypic males and 10 phenotypic females. Direct PCR product sequencing was interrupted by the presence of a polymorphic imperfect pentanucleotide repeat $\left(\mathrm{ACCCC}_{n}\right)$ in the intronic region between exon 3 and exon 4 (data not shown). The successfully sequenced regions comprised between $9.6 \%$ and $94.4 \%$ of the amplicon, although those regions uniformly had a high percent identity with the rspo1 genomic reference $(98.1 \%$ - 100\% pairwise percent identity) from two fully sequenced BAC clones (Pv100_O13 and Pv215_D15). To circumvent this problem we cloned and sequenced PCR products and confirmed the rspol sequence identity for the full length of the amplicon (Additional file 3).

\section{Mapping rspo 1}

To determine if rspo1 lies with oprd1 and rcc1 on the sex chromosomes in P. vitticeps, we mapped two BAC clones (Pv100_O13 and Pv215_D15) containing rspo1. Each BAC clone was physically mapped on to $P$. vitticeps metaphase chromosome spreads along with a $P$. vitticeps sex chromosome specific BAC clone, Pv03_L07 (Figure 3a,b). These two colour FISH experiments (Pv03_L07 + Pv100_O13 and Pv03_L07 + Pv215_D15) showed hybridisation signals on the centromeric region of the $\mathrm{W}$ chromosome in females (yellow signal in Figure 3a,b), as well as a single pair of microchromosomes in both males and females (Figure 3a,b, only female patterns are presented in Figure 3).

Since this pattern could have been produced by shared repetitive sequences, we carried out a second round of FISH with the rspo1 positive BAC clone Pv100_O13, the sex chromosome BAC clone Pv03_L07, and rspo1 cDNA clone (GenBank Accession: KF541659) on the same chromosome preparations. The cDNA clone mapped only a pair of microchromosomes (Figure 3d). Because of the small size of the cDNA clone (about $1.3 \mathrm{~kb}$ ), the hybridisation signal strength on chromosomes was weak and the frequency of hybridisation signals per cell was also low. Only about 30\% metaphase cells had signals and signals were usually on only one chromosome, which is usually common in FISH mapping of cDNA clones [50]. The autosomal microchromosome to which rspo1 cDNA clones mapped was identified by co-hybridisation with BAC clone Pv100_O13. No metaphase cell had rspo1 cDNA signals on sex microchromosomes identified by clone Pv03_L07 (Figure 3e). Thus evidence from the hybridisation of cDNA clone points to rspo1 residing on an autosomal microchromosome pair and not on the sex chromosomes. In addition, we performed two colour FISH using two rspo1 BAC clones (Pv215_D15 + Pv100_O13) with male P. vitticeps' specific c0t-1 DNA as suppressor DNA. Both BAC clones were hybridised onto the same pair of microchromosomes identifying their colocations (Figure 3c). We did not observe any centromeric hybridisation signals on the W chromosomes, suggesting repetitive sequences were successfully suppressed by c0t-1 DNA, which also confirm the cDNA clone mapping, i.e. rspo1 is autosomal. 

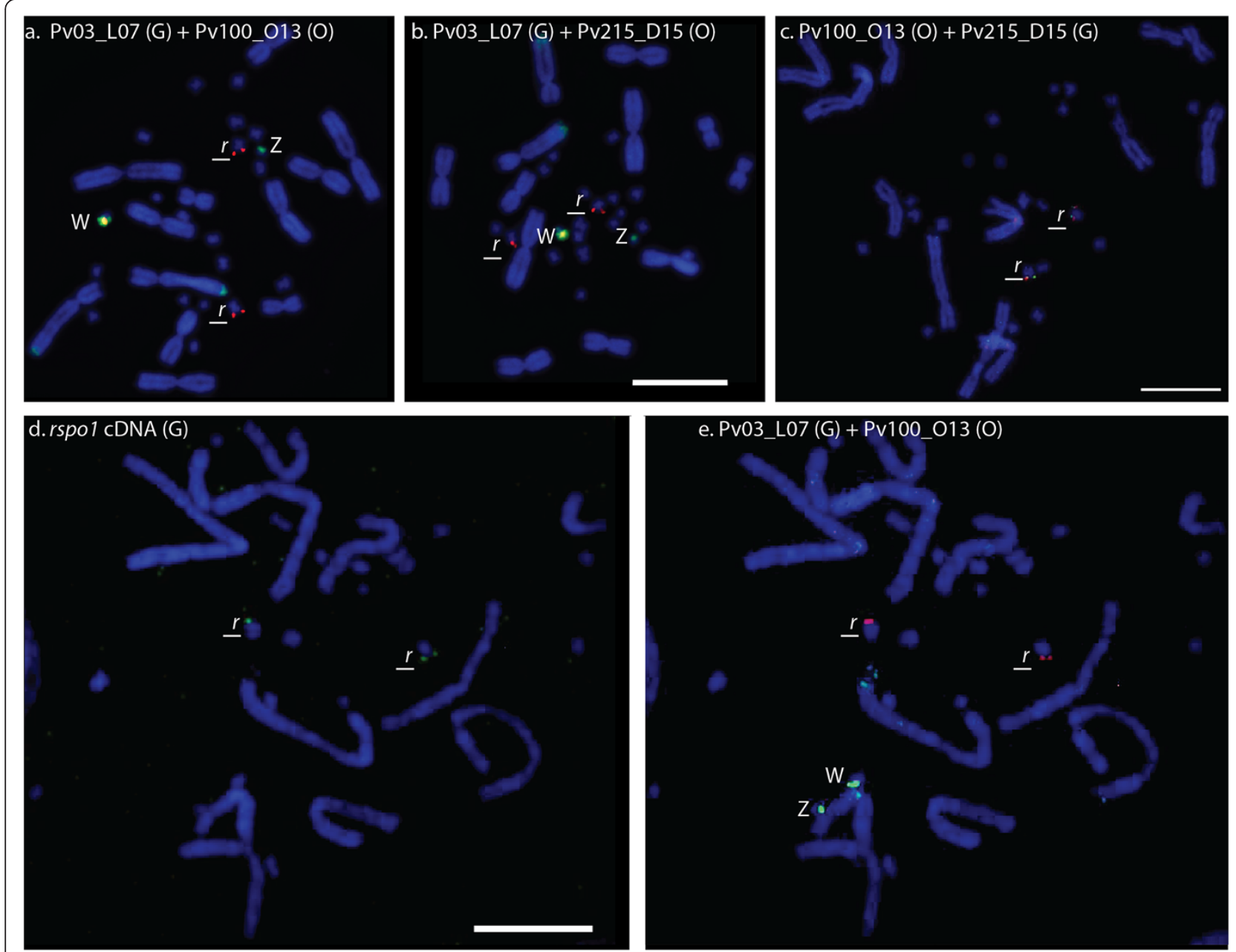

Figure 3 Physical mapping of BAC and CDNA clones containing rspo1 gene in female Pogona vitticeps. a: merged image showing locations of BAC clones Pv03_L07 (green) and Pv100_O13 (orange) containing rspo 1; highly amplified signals from the sex microchromosome BAC clone Pv03_L07 was observed on the W microchromosome, while clear signals from both rspo 1 BAC clones were observed on a different pair of microchromosomes. Note that a yellow signal (overlap of orange and green fluorochromes) is observed on W chromosome, suggesting hybridization of shared repetitive sequences from both BAC clones containing sex chromosome sequences and rspol gene sequences. BAC clone Pv03_L07 hybridised onto $P$. vitticeps sex chromosomes and telomeric region of long arm of chromosome 2; $\mathbf{b}$ : merged image showing locations of BAC clones Pv03_L07 (green) and PV215_D15 (orange) containing rspo1, which had the same pattern as the BAC clone Pv100_O13 (Figure 3a); c: merged image of two rspo1 BAC clones Pv215_D15 (green) and Pv100_O13 (orange), showing co-locations of both BAC clones on the same pair autosomal microchromosomes. Note the absence of hybridisation signal on the $W$ chromosome $(3 a, b)$ which was suppressed by species specific c0t-1 DNA d: hybridization signal of rspolcDNA clone in female metaphase chromosome spreads; e: two colour FISH on the same slide as panel d, showing mapping of P. vitticeps' sex chromosome BAC clone Pv03_L07 (green) with BAC clone Pv100_O13 (orange) containing rspo 1 . Hybridisation signals of sex chromosome BAC clone and the rspol CDNA clone are not on the W chromosomes, suggesting that $r s p o 1$ is autosomal in P. vitticeps. The hybridization signal on the $\mathrm{W}$ chromosome from BAC clone FISH is a result of hybridization of shared repeat sequences contained in those $\mathrm{BAC}$ clones. $\mathrm{G}=$ green; $\mathrm{O}=$ Orange; $r=r$ spo 1; $\mathrm{W}=\mathrm{W}$ chromosome; $\mathrm{Z}=\mathrm{Z}$ chromosome. Scale bars represent $10 \mu \mathrm{m}$.

\section{Rearrangement of the rcc1-oprd1-rspo1 region in Pogona vitticeps}

A closer examination of the region encompassing $r c c 1$, oprd1 and rspol indicated that this region was rearranged during amniote evolution. Although the gene content of this region is conserved between human chromosome 1 and chicken chromosome 23, the Genomicus database [51] has placed $r c c 1$ and oprd1 on a putative amniote ancestral block different from that harboring rspo1. A number of different breakpoints are observed in the rcc1/oprd1 region when gene arrangements of the predicted ancestral blocks are compared to human chromosome 1 and chicken chromosome 23 (Figure 4).

The two BAC clones containing rspo1 (Pv100_O13 and Pv215_D15) also contained 10 genes from the region surrounding RSPO1 in other amniote species, but the sequences from these two BACs do not assemble 


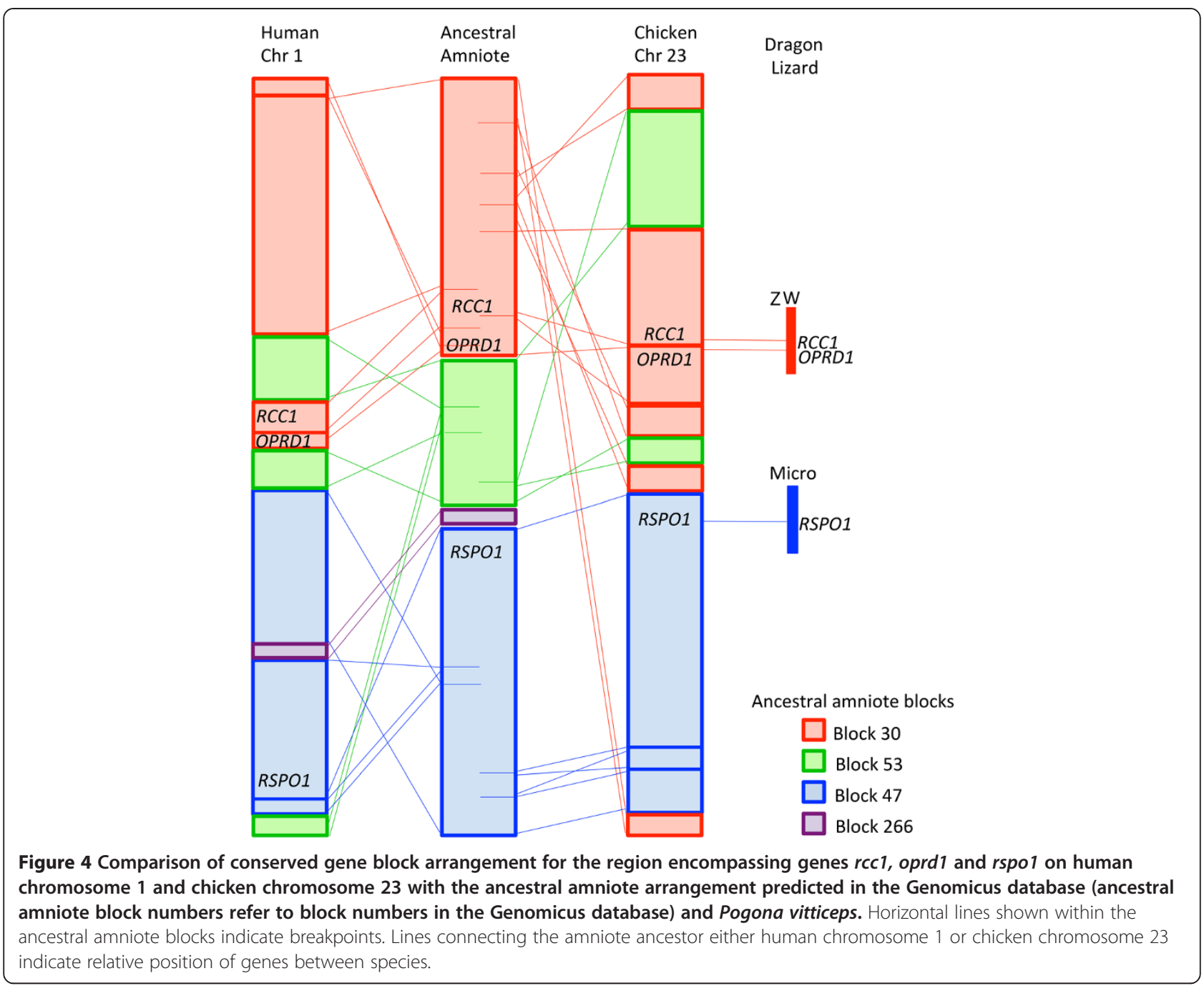

well enough to determine whether gene arrangement is also conserved between $P$. vitticeps and other amniotes.

\section{Discussion}

Our study has characterized the largest contiguous stretch of physically mapped sex chromosome sequence (352 kb) from a ZZ/ZW lizard species. Although this region represents only a small fraction of the sex chromosomes of $P$. vitticeps, it has revealed several features typically associated with sex chromosomes including the accumulation of large blocks of repetitive sequences.

The $352 \mathrm{~kb}$ of sex chromosome sequence that we have identified in this study showed large fold accumulation of LTR and non-LTR retrotransposons including CR1, and LINEs (including BovB, L1, L2). Accumulation of such repetitive sequences are common features on sex chromosomes and have been reported for the sex chromosomes of many vertebrate species from insects to mammals [52-60]. In particular, these repeats have been found to be preferentially accumulated on the sex chromosomes with BovB and CR1 repeats found on the mouse Y chromosomes and the chicken $\mathrm{Z}$ chromosomes respectively. It appears likely therefore, that these repeats may have converged, preferentially on the sex chromosomes in a number of taxa [33] perhaps acting as an evolutionary driver for the degeneration of sex chromosomes in vertebrates.

Our sequencing and physical mapping strategy revealed two known protein coding genes in addition to three zinc finger genes, within the sequence on the $P$. vitticeps sex chromosomes. The gene oprd1 is a member of G-protein-coupled receptors, which constitute a vast protein family that performs a wide range of functions (including various autocrine, paracrine and endocrine processes) $[61,62]$. While the gene $r c c 1$ is a eukaryotic protein which binds to chromatin and interacts with RAN enzyme, a nuclear GTP-binding protein, to promote the loss of bound GTP and the uptake of fresh GTP, thus acting as 
a guanine-nucleotide dissociation stimulator (GDS) [63]. The interaction of $r c c 1$ with RAN probably plays an important role in the regulation of gene expression. Neither of these two genes is known to be involved with sex determination or gonad differentiation of other species and we have no direct evidence that oprd1 and $r c c 1$ are involved in the $P$. vitticeps sex determination pathway. Both are expressed in a range of adult mammalian tissues (NCBI UniGene, [64]). Thus these genes, though on the sex chromosomes of Pogona vitticeps, are unlikely candidates for a sex determination function.

Orthologues of oprd1 and rcc1 are located on a syntenic block that is conserved among amniotes (Figure 4). This synteny suggested an evolutionarily conserved arrangement, which would allow us to identify other genes on the $P$. vitticeps sex chromosomes. One of the genes in this conserved syntenic block, rspo1, is involved in ovary differentiation in mammals. This gene was discovered by its mutation in a family with four XX males, and appears to work by activating beta-catenin pathway $[43,65]$. It was therefore a good candidate for a role in gonad differentiation in $P$. vitticeps. However, it is unlikely to be a candidate for the master sex determining switch as our cDNA clone and two colour BAC FISH mapping has unequivocally established its location on a single pair of autosomal microchromosomes. Although, BAC clones containing rspo1 sequence mapped to the W chromosome as well as an autosomal microchromosome, this is presumably because they contain repeats shared with the sex chromosomes, which can be suppressed by species specific suppressor DNA (Figure 3c). The absence of rspol from the sex chromosomes eliminates this candidate gene from consideration. Importantly, comparative sequence analysis of this newly characterized $P$. vitticeps partial sex chromosome sequence showed no homology to genes or sequence on other vertebrate sex chromosome, implying that the ZW sex chromosomes in this species evolved independently [35].

Evolution of the dragon sex chromosomes seems to have involved genome rearrangements (Figure 4). The $352 \mathrm{~kb}$ contig contains only two genes (oprd1 and rcc1) corresponding to chicken chromosome 23, and they are surrounded by zinc finger genes, which are not present in other amniote species. Intriguingly, $P$. vitticeps is not the only species for which a gene from the ancestral amniote specific synteny block containing oprd1 and $r c c 1$ has ended up on a sex chromosome. For example, med18, a gene adjacent to $r c c 1$ in the predicted ancestral block, is located on the chicken $\mathrm{Z}$ chromosome. Is this merely coincidence, or is there a sequence in this region that has a selectable function in sex determination? In contrast, BAC clones containing rspo1 contain a number of genes found adjacent to this gene in other species. We predict that most of the chicken chromosome 23 synteny block will reside on the same microchromosome pair in P. vitticeps as rspol and not on the sex chromosomes.

\section{Conclusions}

Our study has demonstrated that the sex chromosomes of $P$. vitticeps share some characteristics of sex chromosomes observed in other vertebrate taxa, namely an accumulation of repetitive elements. However the $P$. vitticeps sex chromosomes also revealed new aspects of sex chromosome evolution that may inform us generally about the rapid evolution of novel sex chromosomes. Of particular interest is our observation that a highly conserved syntenic region that contains rspol was disrupted prior to the formation the $P$. vitticeps sex chromosomes. This work provides the fundamental basis and anchor point from which we can walk to characterize the rest of the dragon W chromosome, and discover a putative sex determining gene(s).

\section{Methods}

Isolation and sequencing of BAC clones

A genomic BAC library $(6.2 \mathrm{X})$ for $P$. vitticeps was screened using an overgo-based protocol $[35,66]$. Overgos were designed against previously identified sexlinked sequences (GenBank Accessions: EU938138.1; EU938139.1; EU938136.1), which were extended from a 50 and a $49 \mathrm{bp} \mathrm{W}$ and Z-linked AFLP fragment (Figure 1 for BAC ID) [28,29]. Initial library screening identified five putative sex linked BAC clones. Five more putative sex-linked BAC clones were identified by subsequent BAC walking. We have also used 7-plate PCR matrix of $\mathrm{BAC}$ library to isolate BAC clones containing rspo1, following manufacturer's instruction (Amplicon Express, USA). The library was screened using primer pairs (forward: 5' CAACTGTGAGGACTGTTTCAGC 3'; reverse: 5' AAATCACCTGCAGGATCCAC 3') designed against a testis transcriptome ( $\mathrm{T}$. Ezaz unpublished). Primers were designed to amplify a short genomic fragment between rspol exons 4 and 5 of this gene in P. vitticeps. The PCR conditions was as follows: an initial denaturation at $95^{\circ} \mathrm{C}$ for $5 \mathrm{~min}$, followed by 35 cycles of $95^{\circ} \mathrm{C}$ for $30 \mathrm{~s}, 62^{\circ} \mathrm{C}$ for $30 \mathrm{~s}$ and $72^{\circ} \mathrm{C}$ for $90 \mathrm{~s}$; and finally $72^{\circ} \mathrm{C}$ for $10 \mathrm{~min}$ for a final extension.

The full BAC sequences and BAC end sequences were obtained through a commercial vendor (Macrogen, Korea) and Biomolecular Resource Facility (Australian National University, Canberra, Australia) using Sanger and 454 sequencing platforms. In total five sex chromosome BAC clones and three autosomal BAC clones were sequenced (Table 1) using either Sanger or 454 platform. BAC clones were also subjected to end sequencing using pCC1 epicenter vector specific forward and reverse primers: $\mathrm{pCC1}^{\mathrm{Tm}} / \mathrm{pEpiFOS}^{\mathrm{Tm}}$ forward sequencing primer (5' GGATGTGCTGCAAGGCGATTAAGTT 
GG $3^{\prime}$ ) and $\mathrm{pCC}^{\mathrm{mm}} / \mathrm{pEpiFOS}^{\mathrm{mm}}$ reverse sequencing Primer (5' CTCGTATGTTGTGTGGAATTGTGAGC 3'; Invitrogen). We assembled each BAC using the default parameters of Newbler (V2.6, Roche CT, USA), with an expected coverage of $40 \mathrm{X}$ and a custom filtering database that contained the BAC vector and host genome (DH10B). Sequences shared by the individually assembled BAC clones indicated that they were contiguous, so we repeated the assembly with reads from all five BAC clones and mapped reads back to the resulting $352 \mathrm{~kb}$ contig. Reads from each BAC occupied a discrete segment of the contig in proportion to the size of the insert, as determined by individual BAC assemblies. Sequences were annotated using Genscan [46] and predicted ORFs then subjected to homology search using Blastp (refseq A. carolinensis [47]). The $352 \mathrm{~kb}$ contig was also analyzed for homology using Blastn (nr).

\section{Fluorescence in situ hybridisation (FISH) BAC clone FISH}

The chromosomal locations of isolated sex chromosomal BAC clones were verified by physical mapping using FISH following protocols described in Ezaz et al. [35]. Briefly, 0.5-1 $\mu \mathrm{g}$ of BAC DNA was labeled by nick translation incorporating directly labeled orange or green dUTP (Abbott Molecular, Botany, NSW, Australia). The labeled BAC DNA was ethanol precipitated, resuspended in $20 \mu \mathrm{l}$ hybridisation buffer (50\% formamide, $10 \%$ dextran sulfate, $2 \mathrm{X}$ SSC, $40 \mathrm{mmol} / \mathrm{L}$ sodium phosphate $\mathrm{pH} 7.0$ and $1 \mathrm{X}$ Denhardt's solution), denatured at $68.5^{\circ} \mathrm{C}$ and hybridised onto metaphase chromosomes spreads overnight at $37^{\circ} \mathrm{C}$. Slide washing and microscopy was performed following the protocol described previously by Ezaz et al. [35].

\section{Fiber FISH}

Fiber FISH was performed following a protocol described by Heng et al. [67] with modifications to further understand the repeat contents of these BAC clones as well as their arrangements on the sex chromosomes. Briefly, chromatin fiber was obtained by treatment with lysis buffer (0.5\% SDS, $50 \mathrm{mM}$ EDTA, $200 \mathrm{mM}$ Tris- $\mathrm{HCl} \mathrm{pH}$ 7.4). The cell suspension was dropped on slides and air-dried. Slides were soaked in lysis buffer at $90-120$ s and quickly lifted out to stretch the chromatin fibers and air-dried. After air-drying, the slides were fixed and dehydrated through an ethanol series. The slides were denatured by treatment with 70\% $0.1 \mathrm{~N} \mathrm{NaOH}, \mathrm{EtOH} 30 \%(\mathrm{v} / \mathrm{v})$ for $90 \mathrm{~s}$ at room temperature. Probe hybridisation, detection and slide analysis was performed following the standard FISH protocol described above.

\section{CDNA clone FISH}

We cloned the cDNA of a homologue of rspo1 in $P$. vitticeps. Total RNA was extracted from testicular tissue using RNeasy Plus Universal Mini Kit (Qiagen). The cDNA was synthesized by RT-PCR using Oligo (dT)20 Primer and SuperScript III First-Strand Synthesis System (Invitrogen, Australia), and was used as the PCR template to amplify the homologue of rspo1. Two cDNA fragments of the gene were amplified by PCR with the following primer pairs designed against a P. vitticeps' testes transcriptome: F1, 5'-AGACAAGCAAGCCAGCAA AC-3'; R1, 5'-CGGACAAGAGGGTAAGCAGA-3'; F2, 5' -CATCCTAGGAGCAGGGCTGT-3'; R2, 5' -CTGGC CACGTCCTTACTG G-3'. The PCR conditions was as follows: an initial denaturation at $95^{\circ} \mathrm{C}$ for $5 \mathrm{~min}$, followed by 35 cycles of $95^{\circ} \mathrm{C}$ for $30 \mathrm{~s}, 55^{\circ} \mathrm{C}$ for $30 \mathrm{~s}$ and $72^{\circ} \mathrm{C}$ for $90 \mathrm{~s}$; and finally $72^{\circ} \mathrm{C}$ for $10 \mathrm{~min}$ for a final extension. The PCR products were cloned using TOPO TA Cloning Kit (Invitrogen). We sequenced three cDNA clones for respective fragments from a commercial vendor (Macrogen, Korea).

FISH for cDNA clones was performed as described previously by Matsuda et al. [50] with slight modification. The DNA probes were labeled using nick translation kit (Roche Diagnostics) incorporating biotin-16-dUTP according to standard protocol. Labeled probes were purified by ethanol precipitation, mixed with hybridisation buffer (50\% formamide, 2X SSC, $10 \%$ dextran sulfate, $1 \mathrm{mg} / \mathrm{ml} \mathrm{BSA})$ and denatured by incubation at $75^{\circ} \mathrm{C}$ for $10 \mathrm{~min}$. Chromosome slides were denatured in $70 \%$ formamide $(\mathrm{v} / \mathrm{v}) / 2 \mathrm{X}$ SSC for $2 \mathrm{~min}$ at $70^{\circ} \mathrm{C}$. Approximately, $250 \mathrm{ng}$ of labeled probe $(20 \mu \mathrm{l})$ per slide was hybridised onto metaphase chromosomes for 1 day at $37^{\circ} \mathrm{C}$. Post-hybridisation washes were carried out as following: $50 \%$ formamide $(\mathrm{v} / \mathrm{v}) / 2 \mathrm{X}$ $\mathrm{SSC}$ for $20 \mathrm{~min}$ at $37^{\circ} \mathrm{C}, 2 \mathrm{X} \mathrm{SSC}$ for $15 \mathrm{~min}$ at room temperature, $1 \mathrm{X} \mathrm{SSC}$ for $15 \mathrm{~min}$ at room temperature and 4X SSC for $5 \mathrm{~min}$ at room temperature. The hybridised cDNA probes were reacted with goat anti-biotin antibodies (Vector Laboratories) diluted in 1\% BSA/4X SSC for $1 \mathrm{~h}$ at $37^{\circ} \mathrm{C}$. The chromosome slides were washed as follows: $4 \mathrm{X}$ SSC for $5 \mathrm{~min}$ at room temperature, 0.1\% IGEPAL/4X SSC for $5 \mathrm{~min}$ at room temperature, and $4 \mathrm{X} \mathrm{SSC}$ for $5 \mathrm{~min}$ at room temperature. Then the hybridised probes were reacted with Alexa488 rabbit anti-goat IgG (Invitrogen). The chromosome slides were washed using the following series; 4X SSC for $10 \mathrm{~min}$ at room temperature, $0.1 \%$ IGEPAL/ $4 \mathrm{X}$ SSC for $10 \mathrm{~min}$ at room temperature, and $4 \mathrm{X}$ SSC for $10 \mathrm{~min}$ at room temperature. The slides were counter-stained with $20 \mu \mathrm{g} / \mathrm{ml}$ DAPI in 2X SSC and mounted with VectaShield (Vector Laboratories).

\section{PCR amplification, cloning and sequencing of rspo 1 from multiple Pogona vitticeps individuals}

To determine the level of polymorphisms at the sequence level between male and female $P$. vitticeps, we screened 20 individuals (10 phenotypic males and 10 phenotypic females). We used the same primer pair to 
screen the BAC library and followed the same PCR condition. We cloned eight PCR fragments from three males and three females using pGEM-T Easy vector Systems, (Promega) and TOP10 competent cells (Invitrogen). We sequenced the PCR products using M13 and M13R primers using a commercial vendor (Macrogen, Korea).

\section{Availability of supporting data}

All the supporting data are included as additional files.

\section{Additional files}

\section{Additional file 1: Gene content of three autosomal BAC clones. Sequences were annotated using Genscan [46] and homology search was performed using Blastp [47].}

Additional file 2: Scalable vector graphics (SVG) plot of five sex chromosome and three autosome BAC clones generated by Repbase [48] showing the distribution of repetitive sequences. This figure highlights high frequency of red vertical bars representing locations of repetitive sequences in sex microchromosomes compared to low frequencies of red vertical bars on autosomes, which are also microchromosomes.

Additional file 3: Sequence alignment of cloned PCR amplified rspo 1 fragments from three males and females and two BAC clones containing rspo 1, showing the location of primers and occasional SNPs which are not sex-linked.

\section{Abbreviations}

TSD: Temperature-dependent sex determination; GSD: Genotypic sex determination; ORF: Open reading frame; Overgo: Overlapping oligonucleotide probe; BAC: Bacterial artificial chromosome;

FISH: Fluorescence in situ hybridisation; Contig: Contiguous assembled sequence; cDNA: Complementary DNA; AFLP: Amplified fragment length polymorphism; SNP: Single nucleotide polymorphism; LINE: Long interspersed nuclear element; CR1: Chicken repeat 1 (LINE); GTP: Guanosine5'-triphosphate; GDS: Guanine-nucleotide dissociation stimulator; BLAST: Basic Local Alignment Search Tool; STR: short tandem repeat (microsatellite).

\section{Competing interests}

The authors declare that they have no competing interests.

\section{Authors' contributions}

The authors have made the following declarations about their contributions: Conceived, designed and directed the experiments: TE, SDS, AG, JMG. Performed the experiments: TE, DOM, MY, BA, KM, XZ, MJE Contributed analysis tools: TE, AG, DOM, JD, CH; Wrote the paper: TE, JMG, AG, JD, SDS. All coauthors contributed in reviewing the paper. All authors read and approved the final manuscript.

\section{Funding}

This work was funded by ARC Discovery Grants awarded to SDS, AG and SVE (DP0881196), and to TE, SDS, AG, JMG and Yoichi Matsuda (DP110102262). TE and JD are supported by ARC Future Fellowships (FT110100733, FT100100241). We would like to thank Jacqui Richardson and Alistair Zealey for their care of captive animals and Anna Macdonald for initial discussion about microsatellite mining. All experiments were performed with the approval of the University of Canberra Animal Experimentation Ethics Committee.

\section{Author details}

'Institute for Applied Ecology, University of Canberra, Canberra 2601, Australia. ${ }^{2}$ La Trobe Institute of Molecular Biology, La Trobe University, Melbourne, VIC 3086, Australia. ${ }^{3}$ Department of Organismic and Evolutionary Biology, Harvard University, 26 Oxford Street, Cambridge, MA 02138, USA.

Received: 19 August 2013 Accepted: 13 December 2013

Published: 17 December 2013

\section{References}

1. Takagi N, Sasaki M: A phylogenetic study of bird karyotypes. Chromosoma 1974, 46:91-120.

2. Pokorna M, Giovannotti M, Kratochvil L, Kasai F, Trifonov VA, O'Brien PC, Caputo V, Olmo E, Ferguson-Smith MA, Rens W: Strong conservation of the bird $Z$ chromosome in reptilian genomes is revealed by comparative painting despite 275 million years divergence. Chromosoma 2011, 120:455-468.

3. Pokorna M, Giovannotti M, Kratochvil L, Caputo V, Olmo E, Ferguson-Smith MA, Rens W: Conservation of chromosomes syntenic with avian autosomes in squamate reptiles revealed by comparative chromosome painting. Chromosoma 2012, 121:409-418.

4. Ezaz T, Stiglec R, Veyrunes F, Graves JAM: Relationships between vertebrate ZW and XY sex chromosome systems. Curr Biol 2006, 16:R736-743.

5. Sarre SD, Ezaz T, Georges A: Transitions between sex-determining systems in reptiles and amphibians. Annu Rev Genomics Hum Genet 2011, 12:391-406.

6. Ezaz T, Sarre SD, O'Meally D, Graves JA, Georges A: Sex chromosome evolution in lizards: independent origins and rapid transitions. Cytogenet Genome Res 2009, 127:249-260.

7. Janes $\mathrm{DE}$, Organ $\mathrm{CL}$, Edwards SV: Variability in sex-determining mechanisms influences genome complexity in reptilia. Cytogenet Genome Res 2009, 127:242-248

8. Janes DE, Organ CL, Fujita MK, Shedlock AM, Edwards SV: Genome evolution in Reptilia, the sister group of mammals. Annu Rev Genomics Hum Genet 2010, 11:239-264.

9. Fisher RA: The Evolution of Dominance. Biol Rev 1931, 6:345-368.

10. Charlesworth B: The evolution of sex chromosomes. Science 1991, 251:1030-1033.

11. Charlesworth $B$, Charlesworth D: The degeneration of $Y$ chromosomes. Philos T R Soc B 2000, 355:1563-1572.

12. Nei M: Linkage modifications and sex difference in recombination. Genetics 1969, 63(3):681-699.

13. Bull Jj: Evolution of sex determining mechanisms. Menlo Park, California: Benjamin/Cummings; 1983

14. Rice WR: Evolution of the $Y$ sex chromosome in animals. Bioscience 1996, 46:331-343.

15. Graves JAM: Sex chromosome specialization and degeneration in mammals. Cell 2006, 124:901-914.

16. Henking H: Über Patagonian pejerrey (Odontesthes hatcheri) spermatogenese und deren Beziehung zur Entwicklung bei Pyrrhocoris apterus L. Zeitschrift für wissenschaftliche Zoologie 1891, 51:685-736.

17. Stevens NM: Studies in spermatogenesis, with especial reference to the "accessory chromosome". Carnegie Inst Wash 1905, 36:33.

18. Wilson EB: The Chromosomes in Relation to the Determination of Sex in Insects. Science 1905, 22:500-502.

19. Sinclair AH, Berta P, Palmer MS, Hawkins JR, Griffiths BL, Smith MJ, Foster JW, Frischauf AM, Lovell-Badge R, Goodfellow PN: A gene from the human sex-determining region encodes a protein with homology to a conserved DNA-binding motif. Nature 1990, 346:240-244.

20. Koopman P, Munsterberg A, Capel B, Vivian N, Lovell-Badge R: Expression of a candidate sex-determining gene during mouse testis differentiation. Nature 1990, 348:450-452

21. Matsuda M, Nagahama Y, Shinomiya A, Sato T, Matsuda C, Kobayashi T, Morrey CE, Shibata N, Asakawa S, Shimizu N, et al: DMY is a Y-specific DM-domain gene required for male development in the medaka fish. Nature 2002, 417:559-563.

22. Nanda I, Kondo M, Hornung U, Asakawa S, Winkler C, Shimizu A, Shan Z, Haaf T, Shimizu N, Shima A, et al: A duplicated copy of DMRT1 in the sex-determining region of the $Y$ chromosome of the medaka, Oryzias latipes. Proc Natl Acad Sci USA 2002, 99:11778-11783.

23. Myosho $T$, Otake $H$, Masuyama $H$, Matsuda M, Kuroki $Y$, Fujiyama $A$, Naruse K, Hamaguchi S, Sakaizumi M: Tracing the emergence of a novel sex-determining gene in medaka, Oryzias luzonensis. Genetics 2012, 191:163-170.

24. Yoshimoto S, Ito M: A ZZ/ZW-type sex determination in Xenopus laevis. FEBS J 2011, 278:1020-1026.

25. Yano A, Guyomard R, Nicol B, Jouanno E, Quillet E, Klopp C, Cabau C, Bouchez O, Fostier A, Guiguen Y: An immune-related gene evolved into 
the master sex-determining gene in rainbow trout, Oncorhynchus mykiss. Curr Biol 2012, 22:1423-1428.

26. Hattori RS, Murai Y, Oura M, Masuda S, Majhi SK, Sakamoto T, Fernandino I, Somoza GM, Yokota M, Strussmann CA: A Y-linked anti-Mullerian hormone duplication takes over a critical role in sex determination. Proc Natl Acad Sci USA 2012, 109:2955-2959.

27. Radder RS, Quinn AE, Georges A, Sarre SD, Shine R: Genetic evidence for co-occurrence of chromosomal and thermal sex-determining systems in a lizard. Biol Lett 2008, 4:176-178.

28. Quinn AE, Georges A, Sarre SD, Guarino F, Ezaz T, Graves JA: Temperature sex reversal implies sex gene dosage in a reptile. Science 2007, 316:411.

29. Quinn AE, Ezaz T, Sarre SD, Graves JM, Georges A: Extension, single-locus conversion and physical mapping of sex chromosome sequences identify the Z microchromosome and pseudo-autosomal region in a dragon lizard, Pogona vitticeps. Heredity 2010, 104:410-417.

30. Ohno S: Sex Chromosomes and Sex-Linked Genes. Heidelberg, Germany: Springer-Verlag; 1967.

31. Matsuda Y, Nishida-Umehara C, Tarui H, Kuroiwa A, Yamada K, Isobe T, Ando J, Fujiwara A, Hirao Y, Nishimura O, et al: Highly conserved linkage homology between birds and turtles: bird and turtle chromosomes are precise counterparts of each other. Chromosome Res 2005, 13:601-615.

32. Matsubara K, Tarui H, Toriba M, Yamada K, Nishida-Umehara C, Agata K, Matsuda $Y$ : Evidence for different origin of sex chromosomes in snakes, birds, and mammals and step-wise differentiation of snake sex chromosomes. Proc Natl Acad Sci USA 2006, 103:18190-18195.

33. O'Meally D, Patel HR, Stiglec R, Sarre SD, Georges A, Marshall Graves JA, Ezaz T: Non-homologous sex chromosomes of birds and snakes share repetitive sequences. Chromosome Res 2010, 18:787-800.

34. O'Meally D, Ezaz T, Georges A, Sarre SD, Graves JA: Are some chromosomes particularly good at sex? Insights from amniotes. Chromosome Res 2012, 20:7-19.

35. Ezaz T, Moritz B, Waters P, Marshall Graves JA, Georges A, Sarre SD: The ZW sex microchromosomes of an Australian dragon lizard share no homology with those of other reptiles or birds. Chromosome Res 2009, 17:965-973.

36. Kawagoshi T, Uno Y, Matsubara K, Matsuda Y, Nishida C: The ZW micro-sex chromosomes of the Chinese soft-shelled turtle (Pelodiscus sinensis, Trionychidae, Testudines) have the same origin as chicken chromosome 15. Cytogen Genome Res 2009, 125:125-131.

37. Kawai A, Ishijima J, Nishida C, Kosaka A, Ota H, Kohno S, Matsuda Y: The ZW sex chromosomes of Gekko hokouensis (Gekkonidae, Squamata) represent highly conserved homology with those of avian species. Chromosoma 2009, 118(1):43-51.

38. Alfoldi J, Di Palma F, Grabherr M, Williams C, Kong L, Mauceli E, Russell P, Lowe CB, Glor RE, Jaffe JD, et al: The genome of the green anole lizard and a comparative analysis with birds and mammals. Nature 2011, 477:587-591.

39. Ezaz T, Quinn AE, Sarre SD, O'Meally D, Georges A, Graves JA: Molecular marker suggests rapid changes of sex-determining mechanisms in Australian dragon lizards. Chromosome Res 2009, 17:91-98.

40. Ezaz T, Quinn AE, Miura I, Sarre SD, Georges A, Marshall Graves JA: The dragon lizard Pogona vitticeps has ZZ/ZW micro-sex chromosomes. Chromosome Res 2005, 13:763-776.

41. Witten JG: Some karyotypes of Australian agamids (Reptilia: Lacertilia). Austral J Zool 1983, 31:533-540.

42. Young MJ, O'Meally D, Sarre SD, Georges A, Ezaz T: Molecular cytogenetic map of the central bearded dragon, Pogona vitticeps (Squamata: Agamidae). Chromosome Res 2013, 21:361-374.

43. Parma P, Radi O, Vidal V, Chaboissier MC, Dellambra E, Valentini S, Guerra L, Schedl A, Camerino G: R-spondin1 is essential in sex determination, skin differentiation and malignancy. Nature Genet 2006, 38:1304-1309.

44. Chassot AA, Ranc F, Gregoire EP, Roepers-Gajadien HL, Taketo MM, Camerino G, de Rooij DG, Schedl A, Chaboissier MC: Activation of beta-catenin signaling by Rspo1 controls differentiation of the mammalian ovary. Hum Mol Genet 2008, 17:1264-1277.

45. Zhou L, Charkraborty T, Yu X, Wu L, Liu G, Mohapatra S, Wang D, Nagahama Y: $\mathrm{R}$-spondins are involved in the ovarian differentiation in a teleost, medaka (Oryzias latipes). BMC Dev Biol 2012, 12:36.

46. Burge C, Karlin S: Prediction of complete gene structures in human genomic DNA. J Mol Biol 1997, 268:78-94.

47. Gish W, States DJ: Identification of protein coding regions by database similarity search. Nature Genet 1993, 3:266-272.
48. Jurka J, Kapitonov W, Pavlicek A, Klonowski P, Kohany O, Walichiewicz J: Repbase update, a database of eukaryotic repetitive elements. Cytogenet Genome Res 2005, 110:462-467.

49. Faircloth BC: msatcommander: detection of microsatellite repeat arrays and automated, locus-specific primer design. Mole Ecol Res 2008, 8:92-94.

50. Matsuda Y, Chapman VM: Application of fluorescence in situ hybridisation in genome analysis of the mouse. Electrophoresis 1995, 16:261-272.

51. Louis A, Muffato M, Roest Crollius H: Genomicus: five genome browsers for comparative genomics in eukaryota. Nucleic acids Res 2013, 41:D700-705.

52. Ferreira IA, Martins C: Physical chromosome mapping of repetitive DNA sequences in Nile tilapia Oreochromis niloticus: evidences for a differential distribution of repetitive elements in the sex chromosomes. Micron 2008, 39:411-418.

53. Harvey SC, Boonphakdee C, Campos-Ramos R, Ezaz MT, Griffin DK, Bromage NR, Penman P: Analysis of repetitive DNA sequences in the sex chromosomes of Oreochromis niloticus. Cytogenet Genome Res 2003, 101:314-319.

54. Kraemer C, Schmidt ER: The sex determining region of Chironomus thummi is associated with highly repetitive DNA and transposable elements. Chromosoma 1993, 102:553-562.

55. Cioffi MB, Camacho JP, Bertollo LA: Repetitive DNAs and differentiation of sex chromosomes in neotropical fishes. Cytogenet Genome Res 2011, 132:188-194.

56. Hughes JF, Skaletsky H, Brown LG, Pyntikova T, Graves T, Fulton RS, Dugan S, Ding $Y$, Buhay CJ, Kremitzki C, et al: Strict evolutionary conservation followed rapid gene loss on human and rhesus Y chromosomes. Nature 2012, 483:82-86.

57. Charlesworth B: Model for evolution of $Y$ chromosomes and dosage compensation. Proc Natl Acad Sci U S A 1978, 75:5618-5622.

58. Erlandsson R, Wilson JF, Paabo S: Sex chromosomal transposable element accumulation and male-driven substitutional evolution in humans. Mol Biol Evol 2000, 17:804-812.

59. Shedlock AM: Exploring frontiers in the DNA landscape: an introduction to the symposium "Genome Analysis and the Molecular Systematics of Retroelements". Systematic Biol 2006, 55:871-874.

60. Shedlock AM, Botka CW, Zhao S, Shetty J, Zhang T, Liu JS, Deschavanne PJ, Edwards SV: Phylogenomics of nonavian reptiles and the structure of the ancestral amniote genome. Proc Natl Acad Sci USA 2007, 104:2767-2772.

61. Kieffer BL, Befort K, Gaveriaux-Ruff C, Hirth CG: The delta-opioid receptor: isolation of a cDNA by expression cloning and pharmacological characterization. Proc Natl Acad Sci USA 1992, 89:12048-12052.

62. Stevens $C W$ : The evolution of vertebrate opioid receptors. Front biosci 2009, 14:1247-1269.

63. Makde RD, England JR, Yennawar HP, Tan S: Structure of RCC1 chromatin factor bound to the nucleosome core particle. Nature 2010, 467:562-566.

64. Schuler GD: Pieces of the puzzle: expressed sequence tags and the catalog of human genes. J Mol Med 1997, 75:694-698.

65. Kim KA, Zhao J, Andarmani S, Kakitani M, Oshima T, Binnerts ME, Abo A Tomizuka K, Funk WD: R-Spondin proteins: a novel link to beta-catenin activation. Cell Cycle 2006, 5:23-26.

66. Ross M, LaBrie T, McPherson S, Stanton VP: Screening large-insert libraries by hybridisation. In Current Protocols in Human Genetics. New York: John Wiley Sons; 1999:5.6.1-5.6.32.

67. Heng HH, Squire J, Tsui LC: High-resolution mapping of mammalian genes by in situ hybridisation to free chromatin. Proc Nat Acad Sci USA 1992, 89:9509-9513.

\section{doi:10.1186/1471-2164-14-899}

Cite this article as: Ezaz et al:: Sequence and gene content of a large fragment of a lizard sex chromosome and evaluation of candidate sex differentiating gene R-spondin 1. BMC Genomics 2013 14:899. 